\title{
Mereologies as the Grammars of Chemical Discourses
}

\author{
Rom Harré and Jean-Pierre Llored
}

'If you cut a crumb in half do you have two new crumbs or two halves of a crumb?' John Palmer, quoted in the Sunday Times, 28 June 2009, News Review, p. 16.

Since Robert Boyle's corpuscularian philosophy, chemistry has been a mereological science. Displacing the metaphysics of 'continuous substances' and 'qualities' as the expression of "principles", chemistry has been built on a 'part-whole' metaphysics. The grammar for the use of 'part-whole' concepts is mereology. Taking chemistry to be the science of the transformation of substances by the manipulation of their constituent material parts which are also bits of discrete substances, the elements, this science seems to fit the concepts of classical mereology neatly. The scheme has served as a popular and pedagogical foundation for chemical concepts and explanations in traditional chemical discourse. A sodium atom is a part of a molecule of sodium carbonate and also a part of the extended material substance, the element sodium.

However, chemistry has long since ceased to be based on a simple Boylean metaphysics. From a metaphysical point of view the Boylean picture has been subverted by Earley's (2004) arguments in favour of a process metaphysical foundation, which is naturally tied in with such notions as causal powers and affordances. From the point of view of working chemists the appearance of molecular orbitals as an account of the binding processes of molecules also subverts important aspects of Boyle's ontology. In this paper we want to track the developments in chemistry in relation to the presumptions of a variety of mereologies, grammars of chemical discourses, taking account of these developments.

Our argument is based on the identification of variations in both of the 'poles' of the Part - Whole relation.

A. Differences in Wholes:

i. Dissipative wholes in which material constituents change within a stable structure of processes in contrast to wholes in which the parts are material beings self-identical over time. 
ii. Structural wholes in which the parts are components of stable structures in contrast to amorphous wholes.

B. Differences in parts:

i. Those for which criteria of identity are independent of the wholes of which they are parts.

ii. Those for which criteria of identity are conceptually related to the whole of which they are parts.

Instances of chemical discourses in which all four contrasts are salient will be identified and proposals for the mereological principles necessitated will be examined.

\section{Mereologies as Systems of Formal Rules}

The idea that the part-whole relationship was of sufficient importance to warrant a special branch of logic is due to the work of Stanislaus Lesniewski (for Lesniewski's mereology see Simons, 2000: §2.6). Before turning to the recent discussion of the details of the idea that the grammar of discourses concerning chemically relevant substances is mereology, that is implies an ontology of wholes consisting of distinguishable parts, which themselves consist of distinguishable parts, it is worth reminding ourselves of the basic principles of general mereology and sketching some of the debates about the way these principles should be deployed.

\section{Classical Mereological Principles}

Two main mereological principles more or less define the system of mereological rules for discontinuous substances and their parts, in which the whole is uniform, and unstructured. We will refer to this system as the C-mereology.

The Principle of Unique Composition: There is a unique being, the sum or 'fusion' of a certain collection of beings, of which every such being is a part and which has no parts other than such a part. So, for example, a certain actual chemical molecule is a unique collection of just these chemical atoms, and only these chemical atoms. We note that the composition of such a collection does not serve to uniquely identify a molecule as a being of certain kind the properties of molecules include structures as well as components. In practice we need to recognise the difference between 'disparate sums', that is wholes the parts of which 
instantiate different categories or types and uniform sums in which the parts are all of the same category or type. [Axiom MA3 in Simons (2000)].

There are some difficult questions about how category distinctions are managed in mereology. This reminds one of the legendary Japanese tax collector who charged the poor peasants for three items, the bull, the cow and the pair of cattle. We shall call the addition of a whole level concept to the list of parts the 'Japanese tax collector fallacy'.

The Principle of Mereological Transitivity: If $\mathrm{B}$ is a part of $\mathrm{A}$ and $\mathrm{C}$ is a part of $\mathrm{B}$, then $\mathrm{C}$ is a part of A. [Simons (2000), Axiom MA2].

Various exceptions have been offered to this principle. Some turn on the issue of the way a component is a part of the being of which it is a component or part. A gear wheel is a part of a gear box, but is a tooth of that gear wheel a part of the gear box in the same way? If we include function among the attributes that define how a being is a constituent of another being, that is how it is a part, then clearly a tooth is a part of a gear wheel in a different way from the way a gear wheel is part of a gear box, and transitivity of that part-whole relation fails. Each has a quite different functional relationship to the whole of which it is a part. This observation leads on to the need to formulate a second mereology, one in which the principles include structural-functional relations.

\section{Functional Mereological Principles}

Even though constituents lose their actualised functional attributes when removed from the whole of which they have been parts, they do not cease to exist. Nor do they lose the core attributes that enabled them to count as parts of the relevant whole. In the light of our knowledge of how a component fits into a whole we may want to hold that potential functionality survives some ways of decomposing the original whole. For example setting fire to a chair is a mode of decomposition into parts that does not preserved potential functionality.

Consider the parts of a chair - qua material objects but not identified as beings of certain kinds by the criteria of carpenters. They continue to exist and have all their material attributes, size, shape, weight etc as bits of wood when the chair is disassembled.

However, they do not preserve their formerly occurrent functional attributes after disassembly - chair parts move from actual to potential functions, e.g. the seat was actually 
then and there supporting the weight of sitter, but detached from the frame that function is only potential. Turning to an essentially Aristotelian view of the scope of usable categories we note that chemical synthesis creates temporary dispositions that allow chemists to lift the reacting systems over the required energy thresholds. As we shall see, Mullikan's work allows us to maintain the dynamical point of view of the mereology we will be developing in this paper.

This aspect of wholes has been discussed by Rescher and Oppenheim aeons ago (1955). They suggest three conditions on wholes: a whole must possess an attribute that is peculiar to it as a whole; the parts of a whole must stand in some special relationship to one another; a whole must have a structure.

The above analysis seems to presuppose the concept of an emergent property fully to describe the whole of which functionally specified components are parts. To ascribe a function to a chair leg makes sense only if the assemblage of chair parts has a structure which endows these parts as assembled with certain causal powers, such as the ability to support the weight of a person. In general, emergent properties do not satisfy the mereological principle of transitivity.

\section{Set theoretical Mereological Principles.}

Lewis begins his sketch of the basic principles of set theoretical mereology with an example to illustrate the concept of 'fusion' and 'sum'. It falls somewhere between the examples of continuous and discontinuous wholes above. 'The fusion of all cats is that large, scattered chunk of cat-stuff which is composed of all the cats there are, and nothing else' (Lewis, 1991: 1). Neither past cats nor future cats are parts of the cat-fusion. Simons's concept of 'fusion' is different from that of Lewis. For Lewis 'sum', that is 'all the cats', is the same as 'fusion'. For Simons some bunch of cats taken as a whole is a fusion, though it may not include all the cats. So there may be several cat-fusions. Assuming transitivity Lewis remarks that the parts of cats are also parts of the cat-fusion. This allows Lewis to distinguish the class of cats from the fusion of cats - the mereological attributes of lots of cats from their set theoretical attributes. Since the member of a member of a set is not in general a member of that set, membership is not the same relation as part to whole. However, Lewis does allow that classes do have parts, their subclasses (Lewis, 1991: 3). So there is the possibility of a mereologised set theory, or a set-theoretical mereology 
He proposes several mereological principles for sets and their relations to individuals (Lewis, 1991: 7). Using the concept of a 'fusion', Lewis refines the simple Lesniewskian scheme with additional principles for a set theoretical mereology (Lewis, 1991: 74).

1. Transitivity: If $x$ is a part of some part of $y$, then $x$ is a part of $y$.

2. Unrestricted Composition: Whenever there are some things, then there exists a fusion of those things.

3. Uniqueness of Composition: It never happens that the same things have two different fusions.

Then he sets out the way mereological concepts are to be given a set-theoretical interpretation, that is mapped on to the part - whole distinction.

4.One class is a part of another if and only if the first is a subclass of the second.

6. No class has any part that is not a class.

7. Reality divides exhaustively into individuals and classes.

8. No class is part of an individual.

9. Any fusion of individuals is an individual.

Chemists with stereo-isomers, carbohydrates and so on in mind will surely find the principle of uniqueness of composition unintuitive, and inadequate to chemical part-whole reasoning. Lewis's mereology of sets (to be called the S-mereology) sidelines structure and so already leaves itself open to counter-examples to its principles from chemistry.

\section{Choosing a Mereology for Chemical Discourses.}

Chemical discourse is largely based on a distinction, hard won it is true, between elements, compounds and mixtures. It is evidently structured by mereological concepts. Clearly ioncores are parts of elements in different way from that in which they are parts of compounds. Elements are uniform fusions or sums of nuclei atoms, identified by atomic number, $\mathrm{Z}$. Compounds are disparate sums, because in general the constituents of molecules include ioncores from different elements or more exactly from their isotopes as well. Mixtures are also disparate fusions or sums but the parts are not causally related into relatively permanent 
structures, nor is there a determinate proportionality among the parts of a mixture.

But which version of mereology should we prefer? Classical mereology makes use of the lowest level of the current hierarchy of beings in the relations of parts and wholes things, generally spatio-temporally distinct beings with both criteria of numerical identity and of qualitative identity. Two kinds of classical mereological discourses have been distinguished according to whether their several mereological regresses terminate in atoms, in the traditional sense of beings with no proper parts, or do not terminate, every proper part at each level itself having proper parts. A proper part is a part that it not identical to the whole of which it is a part. We have suggested that classical axiomatic mereology can be extended to include rules for the use of a whole - part relation for contexts in which the parts are functionally distinct relative to the whole of which they form parts. In the absence of the concept of the whole, for example a chair, the shapes of the parts, for example, are mereologically irrelevant to their role as parts of that whole. The mereology of functionally defined parts in contrast to an atomic mereology should fit the logic of discourses featuring compounds while atomic mereology should fit the logic of discourses about mixtures.

Moreover, atoms are also parts of distributed elements. Is a sodium atom a part of the element sodium in the way that a horse is part of a herd of horses? Very few elements exist as chunks of well-bounded and uniform stuff - diamonds as chunks of carbon or nuggets as chunks of gold perhaps? This question is made more complicated by Earley's argument in favour of a discourse of 'ion-cores' rather than the atoms of Boyle and Dalton.

Faced with this tidy scheme the question for a philosopher of chemistry is whether the key chemical concepts of substance, element, molecule, atom, subatomic particle, field and so on fit, at least in part, on to the logic of classes, set theory, or on to the principles of classical mereology. Is the element 'sodium' the set of all sodium atoms, or the mereological fusion of all sodium atoms? Is there a mereologised set-theoretical way of distinguishing mixtures from compounds?

What difference would it make which way we jumped to explicate the forms of reasoning available to chemists? Does the chemical fact that the sodium atoms in the universe exist as a fusion which includes chunks of sodium metal and that they also seem to exist in ionic form as a fusion when arranged in a lattice with chloride ions in a lump of common salt throw doubts on the application of Lewis Axiom c to chemistry? 
However, Lewis does introduce an ontologically and mereologically significant concept in the 'singleton', the single membered class. Set theory requires the possibility of empty classes that collectively constitute the null class. This comes about because to specify a class one needs an intension, that is the necessary attributes that identify a class member, and an extension, that is the members that meet that criterion. There can be a class intension that nothing in the real world satisfies. Its extension is null.

Here we have a genuine alternative ontology - are the atomic constituents of molecules single member subsets that are parts of molecular sets?

\section{Mereological Rules for Continuous Substances}

In his recent debate with Joseph Earley, Rodney Needham (2005) sets out two basic mereological principles for discourses about substances that are considered to be continuous they have bits but not parts. A lump of gold, not yet wrought into anything shapely, can have bits lopped of it, but they are not parts of the lump in the sense that the legs are parts of the chair. It makes little sense to ask someone to bring them a part of glass of beer, or of the sea. 'Bring me some sea water' does make sense but it would be very odd to say that what is in the bucket is a part of the sea, though it is!. Using water as an example and despite the force of the vernacular use of the word 'part', Needham offers the following:

1.The distributive condition: Whatever is a 'part' [sample] 'w', taken from a body of water, ' $\mathrm{W}$ ' is also water.

2. The cumulative condition: If two things, say the contents of a pair of buckets, are water, their sum is water.

These principles generalise nicely to other mass substances, such as wood and even fire. This scheme can be interpreted in terms of the S-mereology. If the parts are subsets of the whole as a set, then each bucket of water is a singleton, that is a single membered set. An empty bucket is a logical possibility in the task that the Sorcerer's Apprentice was stuck with - a nothing will not do as a 'part' in the C-mereology, but there is no problem with null sets. The bucket is, as it were, the intension of the singleton, but it has not extension.

Needham's principles do very well with the part-whole relation as it appears in the traditional chemistry of elements. When we turn to compounds, it is reasonable to consider ions as parts of chemical substances, one molecular cluster of ions is enough to have a 
substance on hand, but they satisfy neither the distributive nor the cumulative condition above. Any old sodium ion and any old chloride ion as candidate parts of a whole salt crystal are not salt. Here is the case of the part-whole relation requiring a third ingredient, the right relation between the parts, in order that the criteria of identity for the relevant whole are met. The structure of a molecule is generally a sum of parts which are from different chemical elements. If it is, we seem to be faced with the molecule as a 'disparate sum'. However, if the molecule we are analysing is a diatomic part of an element, for example $\mathrm{H}_{2}$, then the parts are numerically distinct but qualitatively identical. This is a uniform fusion, not a disparate sum..

\section{Progression of the mereological principle in chemistry}

\section{Earley's mereological argument:}

The conundrum first proposed by Joseph Earley - what is the mereology of the compounds which are ionized in solutions? - subverts the simple mereology of things and their parts, that was the model for Boyle's and Dalton's conceptions of elements and compounds, atoms and molecules. Taking this question further raises the question of the mereological status of the constituents of molecules in the light of the molecular orbital theory of Mulliken. This is not the same question as above, since Mulliken queries the very idea of atoms as parts of molecules, whether fully clothed in electrons or as ion-cores.

According to Earley, $\mathrm{Na}^{+}$and $\mathrm{Cl}^{-}$ions are not parts of ionic crystals of salt when that white, crystalline sample of salt has been dissolved. However, their presence in the solution determines that the brine will afford salt as a mass substance on the carrying out of certain operations on sea water, say evaporating it in a salt pan, and not something else. Thus they are at best potential material parts of salt. They are like the legs of chairs in the factory store room.

Here is a problem. $\mathrm{Na}^{+}$is a potentially a part or constituent of a possible salt crystal afforded by a saline solution when it is in the sea. But it is a potential part of component of a possible soap molecule when it is in a solution with $\mathrm{HO}^{-}$ions. However, it is neither a bit of salt nor a bit of soap in the way that the salt in the salt cellar is a bit of salt, or the cake of Imperial Leather by the shower is a bit of soap. Is an $\mathrm{Na}^{+}$ion then a part of sodium? The relativisation of its mereological status to the mass substance it affords does not have an application when the afforded substance is the element of which it is a part. We are not sure what should be said about allotropes. A lump of lamp black is a fusion of carbon 
atoms, but so is a diamond and fullerene, a modern synthesis. The structural difference cannot be a mere ad hoc 'add on' to the mereology. Yet, taking sodium ions one by one, they do not line up as salt constituents and soap constituents. The chemical and physical environment in which these processes occur is crucial to their mereological status as 'parts' of something.

There seem to be 'vertical' (ion cores in molecules) and 'horizontal' (ioncores/atoms in elements) part-whole relations in chemistry. The vertical compositions can be made sense of only if structure is included as an ineliminable aspect of molecular aggregates. 'Part of the element sodium' in what sense? It cannot be in the sense that a horse shoe is a bit of Iron, there being a distinguishable stuff with bits scattered all over the universe. However, Sodium nowhere exists as a stuff. It exists, so far as we know, only as atomic level instances of a type. At this point in the analysis one is reminded of Nelson Goodman's mereological treatment of the relation between instances and the species they instantiate. All the actual horses make up the extended concrete universal which is the totality of horses scattered across the surface of the Earth (1951). However, horses can never merge into a mass of horse stuff in the way that old cars merge into pig iron in a furnace. A herd of horses is not like a block of cast iron. Each horse retains its integrity as an individual, but each old car does not do so in the iron ingot. In classical chemistry the atomic constituents of molecules were mereologically similar to the horses. However, odd things happen to the idea of integrity of being in the context of subatomic physics.

Earley's analysis demonstrates that the working conceptual system of chemistry, with its strong classical mereological underpinnings, does not permit the claim that there is salt in the sea, as an ocean of brine. However, is there a parallel argument to show that there is no sodium or chlorine in a salt crystal? It seems just as convincing.

Returning to Needham's subtle analysis of the logical conditions for the use of mass substance concepts (Needham, 2005: 109 - 112), we note that he makes use of a distinction between a Stoic conception of mixtures, in which constituents retain their identity, and an Aristotelian conception in which a dissolved substance, e.g. salt, is potentially present in the solution as salt, understood as the product of an extraction procedure. Our solution to Earley's paradox in terms of affordances, introduces a concept that occupies a logical slot between the actuality of Stoic substance and the modal possibility or potentiality of Aristotelian substance. Brine affords salt, but salt is not a mereological part of brine, that is 
does not satisfy Lesniewski's axioms. Logically, affordances are attributes, not constituents. Brine contains the constituents of salt, hence it can afford salt, but it does not contain salt in either the Stoic or the Aristotelian conception of a substance as constituent. The store cupboard contains the ingredients for a cake, but it does not contain any actual or potential cakes. The potentiality or cake affordances are not potential cakes. The logical distinction we need is between substances and attributes. Affordances are attributes not substances, so they are not parts of anything.

\section{The argument so far}

Does it make any sense at all to ask if elements and their atomic constituents and molecules and their atomic components could be treated as sets? If so there is room for mereological set theory à la Lewis as an alternative grammar for chemicals discourses.

\section{The Case for S-mereology}

The case for adopting set theory as the Mereology for chemistry begins with the predictions by Odling and Mendeleev (c.f. Scerri, 2008) of the properties of elements yet to be discovered. At the time of their proposals only the intensions of the set of atoms of ekaiodine was available in chemical discourse. The set had a null extension for the users of the grammar appropriate to the situation as it then stood, since the set had no members, and conceivably might never have any. Obviously there cannot be a fusion or a sum of which there are no parts. To talk of eka-iodine in the grammar of classical Mereology made no sense. It does seem to make sense in a discourse in which the parts of sets are subsets.

Are hydrogen and oxygen atoms subsets of the water molecule set? Each water molecule would be a subset of the superset, the stuff water. However, what is the intension of the set of which two sets, a pair of hydrogen atoms and a singleton oxygen atom are the subsets? Well, it is the properties of whatever it takes to be a subset of the set of water molecules that is the water stuff. The hydrogen atoms are members of the set of all hydrogen atoms, while the oxygen atom is a member of the set of all oxygen atoms. Does this have any advantage over the classical mereological grammar? That it does not is evident from a problem which we note in passing but do not address here - is a single $\mathrm{H}_{2} \mathrm{O}$ molecule properly to be called water? Following Needham (2005) we need to introduce mereologies sensitive to scale.

The Case for the C-mereology 
The first argument for the C-mereology depends on the possibility of a whole having emergent properties as a result of some structural invariants. A set only accidentally has structural properties because it is a conceptual object. A whole has structural properties because is a material entity, with real relations between its parts. Sets are held together by similarity relations, not by real relations between the parts of wholes such as material connectivity (the parts of a chair) or causality, the parts of a molecule. A set can have only similar members, while a whole can have dissimilar parts. A set is a logical object while a whole is a material object.

The second argument for C-mereology depends on the criteria for class membership that is the intensionality component of the set concept. If $\mathrm{H}^{+}$and $\mathrm{O}^{2-}$ are subsets of the water molecule set what is their common property that makes them members of this set? It can only be that they are constituents of a water molecule. Hence the S-mereology treatment of chemical unity in multiplicity depends on a $\mathrm{C}$-mereological understanding of the relation between atoms (ions) and the molecules of which they are parts.

In the grammar of classical Mereology, the three atoms are the parts of a water molecule which is their (disparate) fusion or sum. The water in the sea is the mereological fusion of certain water molecules as parts. But it is not the sum of water molecules, which is a being of much greater dimensions being all the water molecules there are. A bucket of brine as a part of the sea is a fusion of the water molecules which are its parts. As Earley has argued the same does not apply to the $\mathrm{Na}^{+}$and $\mathrm{Cl}^{-}$ions in the sea. Here we need to supplement $\mathrm{C}$ mereology with dispositional concepts as illustrated in the simple case of the parts of the chair. The concept of the whole, the chair, cannot be eliminated from the criteria for ascribing dispositional properties to the chair parts.

However, the C-mereology does very well for the grammar of discourses about mixtures. A mixture is a collectivity or aggregate which includes more than one kind of substance. If we allow 'disparate sums' as a legitimate mereological concept then mixtures are clearly sums or fusions. Mixtures rarely have emergent properties. Their constituents are not causally related nor do they have invariant structures. A sack of sand and cement consists of causally unrelated and independent molecules of silicon oxide and calcium carbonate. When water is added complex chemical reactions begin and the mixture ceases to exist. The mortar that comes into being has emergent properties, such as tensile strength, that a scoop of dry sand and cement does not have. A silicon oxide molecule can be a mereological part of a 
bag of sand and cement, but when that bag of cement becomes a part of a block of concrete of which the other part is water, the transitivity principle becomes problematic because none of the silicon oxide molecules have the emergent properties of the mortar. In general, the transitivity principle is in conflict with the appearance of chemically relevant emergent properties.

\section{Mereological Presumptions in the Historical Analysis of the Concept of Molecular Orbital'}

The progression of the grammar of chemical discourse concerning molecules considered with respect to their mereological constituents goes something like this: the classical account of a molecule was of a disparate fusion of atoms sustained by their individual combining power. About a hundred years ago this modulated into the shared electron theory as the source of bonding, with the perfect octet as the grounding concept. Each electron 'orbited' the nucleus of its own atom since it was a defining constituent of that atom. Thus the 'shell' architecture of sodium with its nuclear cluster of protons and neutrons defined the element. So far so Earley-ish. A crystal of salt contains sodium in the same way that a bucket of brine contains salt - that is it affords sodium, for example in Davy-style electrolysis, just as evaporation forces the bucket of brine to afford salt. However the advent of molecular orbital theory, such as that of Mulliken (1981), requires a more radical mereological grammar. Even ion-cores lose their thing-like status. According to Mulliken (Ramsay and Hinze, 1932: 451) 'Attempts to regard a molecule as consisting of specific atoms or ionic units held together by discrete numbers of bonding electrons or electron pairs are regarded [by me] as more or less meaningless'. So, following Mulliken's thought, we would say that there are no atoms in a salt crystal, for the same reason as there is no salt in the sea, though Mulliken's studies began with binding in covalent molecules. Electrons as constituents of molecular orbitals as an image of electronic density energy distributions are not related to the nuclei of constituent atoms, but to duplets, triplets etc., the paired or tripled etc nuclei at the core of the molecule. Formally, molecular orbitals are linear combinations of atomic orbitals, but the atoms that define these wave functions do not actually exist. Mulliken's view seems to be that they are scaffolding to provide the models needed to arrive at the consequential molecular orbitals and to explain molecular spectra. However, the electrolysis of molten $\mathrm{NaCl}$ affords sodium atoms in plenty because it affords the sodium nuclei that are essential to the formation of sodium, atom by atom. There is no sodium in salt, but salt affords sodium. 
In proposing a change of terminology from 'orbit to 'orbital Mulliken (1932)reassigns the electro-mechanical model of the atom as a basis for an explanation of atomic spectra to a heuristic role. We can be quite agnostic about whether electrons are little things whizzing round a nuclear 'sun'. So far Mulliken's proposal upsets simplistic applications of $\mathrm{C}$ or S-metrological rules to 'atomic chemistry'. However, the role of electrons in binding atomic units into molecules, when interpreted within Mulliken molecular orbital approximation (or approach), upsets the mereology of atoms as well. Or to out in another way, this approach undercuts the simplistic idea of atoms as simple constituents of molecules. This seems to open the way for a revised mereology of affordances à la Earley. Molecules afford atoms though atoms are not simple molecular constituents.

Just as 'electron' ceases to be literally the name of a moving body, so 'atom' ceases to be literally the name of a molecular constituent. If molecular spectra can be explained by molecular 'orbitals' then the case is more or less made. Mulliken makes use of the concept of 'atom' in two distinct but linked ways. The concept of 'atom' is a conceptual tool which makes it possible to unify multiple relations betweenempirical data, particularly spectra. Mulliken's diagrams bring about a synthesis of the experimental data and certain theoretical models thanks to the mediator concept of 'atom'. These diagrams allow a great number of forecasts not only about the spectral states of the molecules but also as regards their physical properties. They make it possible moreover to study the formation of molecules without alluding to a supposed intrinsic valency of the atoms.

The same account can be given of the role of the concept of 'electron'. It plays a heuristic role through the concept of binding capacity of electrons. Mulliken makes use of the process of molecular dynamics to try to rationalize molecular reactivity. The heuristic character of the explanations which he proposes is undeniable but it is not all. Using the 'manipulation' criterion on which to base claims for existence Mulliken points out that electrons exist because they can be acted upon by electromagnetic radiation, the interaction having observable consequences (Harré, 1996). An electron has a relational capacity to interact with various nuclei plus other electrons in a molecular orbit. The consequences of acting upon electrons are displayed by means of spectroscopy. Mulliken does not believe in electrons because he seeks a theory of the structure of matter but because they can be acted upon electromagnetically. Such effects at the electron level as lengthening of the internuclear distances in a molecule, change of the angles of connection, evolution of energies of dissociation and so on, have spectroscopic effects. Mulliken is looking for whatever causal 
capacities are at the origin of the molecular phenomena. He tries to quantify the binding capacity of electrons via many spectral studies thanks to the lever of electronic configuration.

Mulliken believed that this binding capacity is related to the stability and the reactivity of the molecules i.e. on their capacity to act on other molecules. He tried to measure the capacity of electrons to be put in relation with nuclei or electrons of other molecules to produce chemical phenomena. Spectroscopy makes it possible for Mulliken to evaluate this reactive capacity of electrons and to propose ways of envisaging the properties of the molecules starting from analogies between the atomic and molecular spectral states. He specifies that (1931): "we should regard a single bonding electron as the natural unit of bonding, an anti-bonding electron as a negative unit".

Using the expression 'diatomic molecule' for such a thing as a molecule of $\mathrm{HCl}$ or $\mathrm{H}_{2}$ suggests that the mereological analysis of these complex entities should lead us to say that the parts of such molecules are hydrogen and chlorine atoms. However, Mulliken's solution to the problem of how atoms are bound into molecules involves electron orbits that are not centred on the nuclei of the constituent atoms. Instead the wave function for an electron becomes molecule centred, the paired nuclei serving as the reference for the model interpretation of the new orbital as a linear function of the wave equations for each electron considered with respect to each of the apparently constituent nuclei. If the criterion of identity for an atom or the ionic residue of such an atom, is the composition of the electron shells then these criteria could not be satisfied by the components of a complex molecule. The relevant nuclei form a doublet which, speaking in the accent of Mulliken, are a unit without parts, using the molecular orbital theory of electrons as the criterion for an individual part. A molecule does not have atoms or ions or even the nuclei of ions as its parts. It does have nuclei duplets however, identified as molecular parts with respect to molecular orbitals. Furthermore, Mulliken's fragment method (1933) emphasizes the arbitrariness of defining the parts. Two fragments can interact provided they have the same kind of symmetry and that the energy gap, measured by spectroscopy, is not too high. For the ethylene molecule " $\mathrm{C}_{2} \mathrm{H}_{4}$ ", he considers two fragments " $\mathrm{CH}_{2}$ " and determines suitable molecular orbital by using the irreducible representations of ethylene. In doing so, he grasps all the characteristics of molecular orbital diagram of the ethylene molecule. The possibility of an experimental support was all the more important as the nature of the initial fragments can change depending on each specific case. To model the molecule $\mathrm{C}_{2} \mathrm{H}_{2}$, Mulliken can just as easily consider a fragment " $\mathrm{C}_{2}$ " and another " $\mathrm{H}_{4}$ " of adapted symmetries. 
We could express this insight in a mereological principle: Constituent atoms of molecules are not parts of those molecules when we look at the total entity in the light of molecular orbitals. Unlike chair parts which preserve their material properties whether in the chair or on the bench. Nor are they parts in the sense that buckets of water are parts of the ocean.

However, parts of chairs, atoms and the contents of buckets of water are extracted from the wholes of which they are parts by some procedure. Looked at from the point of view of the whole, chairs, molecules and oceans afford things; looked at from the point of view of their constituent parts they are potentialities, not the things that are thereby afforded.

A mereological study must take account of the interaction of the whole with its environment and should not artificially isolate it from the external world. Earley's study of a mereology of chemical systems is important because the chemical entities are defined by their capacities to act on their medium. For even thinking the relation between a molecule and its parts, it is necessary to take account of the capacities of this molecule to act on the external world. A chemist generally seeks criteria that can be used to foresee the existence and the geometry of transition states and those of the products given those of the reactants. This is the goal of molecular theories of reactivity. Mulliken proposes static indexes to study molecular reactivity such as the atomic charges for the prediction of ionic or dipolar reactions, others propose the free valence index for radical reactions. Successive approximations justified by their context of use make it possible to include the behavior of the reagents according to various types of control (steric, electric, frontier orbital) expressed by the Klopman-Salem equation to determine the energy of interaction. In parallel, the dynamic approach to reactivity uses indexes which characterize the response of a molecule to the approach of a reagent. An example of dynamic index of reactivity is the $\pi$-electrons localization energy on an atom in a molecule. But those atomic indexes are afforded by the molecular whole interacted with the surroundings.

This capacity to enter into extra-molecular relations makes it possible to study the chemical properties of atomic aggregates but also accounts for the molecular form i.e. of the internal relations between the molecule and its "parts". In addition, the striking analogy between chemical properties and quantum observables is a line of work that could pave the way for a new mereological approach to chemical systems. Are there atoms in a molecule? Does this question admit an answer similar to that brought by Earley concerning salt in sea 
water? In the light of our arguments, it seems that Mulliken and Earley offer parallel arguments. Molecules afford atoms in the context of certain manipulations as studies of molecular reactivity have shown us. The material content of a molecule can only be a fusion of atomic potentials, not of atoms.

Affordances are not simple conditional properties. This is because they incorporate the procedure or method used to display their empirical manifestations.

The conclusion from this analysis is that an 'atom' in the molecular orbital framework of concepts is mereologically like a single sodium ionic core in Earley's sea, that is it affords salt or in appropriate circumstances, soap, as a proper part of a structured whole, or it affords sodium as a widely distributed element.

\section{Continuous and discontinuous substances as wholes}

Since the end of the eighteenth century the idea that chemistry is the study of the qualitative transformations of continuous substances has been displaced until recently by the simple atomic hypothesis. The mereology of continuous substances does not fit the logic of classes and their members, set theory, as worked out mereologically, by David Lewis (1991) for example. Taking the sea as a continuous substance we can say it is a fusion of trillions of buckets full, how many depends on the size of the bucket. It can be considered as a fusion of a dozen or so oceans and seas, or as a fusion of so many drops from an eye dropper and so on. These scale-different fusions illustrate the transitivity of the part-whole relation.

However, atoms of disparate kinds do not make up molecules in the way that members of sets make up sets, though they are the parts of such molecules (neglecting diatomic and polyatomic molecules for the moment). Nor do atoms of the same kind make up elements in that way either, though the stuff, sodium, has sodium atoms as parts, and blocks of it can be kept anhydrously in a vat of paraffin. Horses make up the set of all horses in yet another way, since horses never fuse into larger equine entities in the way that sodium atoms fuse into larger blocks of sodium. Band theory based on quantum chemistry considers a crystal to be a whole in which atoms lose their identity. This opens up yet another dimension in which the Cmereology looks in need of supplementation. So there seem to be two notions of mereological fusion at work in chemistry.

We are forced to conclude therefore that a new set of mereological rules is required for the logic of chemical discourses. It is neither wholly a C-nor wholly an S-mereology. 
The new mereology requires a revision of the basic principles that are definitive of each of the metrologies set out above.

In classical mereology the Principle of Unique Composition runs up against conclusion that the parts of chemical wholes like molecules and atoms are affordances not themselves concrete entities. However, those same atoms which Mulliken's approximation transforms into affordances are the parts of elements as fusions, that is obey the Unique Composition Principle. It seems to us that Transitivity of the Part-Whole Relation as defined in C-mereology does hold because electron affordances are parts of atoms, and atom affordances are parts of molecules, electron affordances are parts of molecules. That is the conclusion to be drawn from Mulliken's demonstration of the power of the molecular orbital set. Nevertheless, transferring the Principle of the Transitivity of the Part-Whole relation to the S-mereology also appears to be viable. An electron-singleton is a subset of a certain set of electrons, just as a neutron-singleton is a subset of a certain set of neutrons and a positron-singleton is a subset of a certain set of positrons, and these sets are subsets of an atom as a set of subatomic objects. An 'atom' is a subset of a set of atoms, that is a molecule, in the same way as its subatomic parts are subsets of itself a set.

Where do the C-mereology and S-mereology part company as grammars for chemical discourses? So far as we can see - nowhere. Provided we are willing to countenance 'disparate sets', membership of which is determined by a quite complex relation between the functionality of parts and the object as superset they comprise we can think with Lesniewski or with Lewis.

\section{Fugitive Constituents and the Parts in Dissipative Structures.}

A candle flame, such as that which was the subject of Faraday's famous lecture, is a dissipative structure because it is continuously self-identical as a sum of processes. There are many molecular level processes which are parts of the macro process, the flame as a bounded dissipative structure. From this point of view the mereology of Faraday's candle flame is unproblematic (Earley. 2005). However, from another point of view, that of ionic cores as constituents of material beings as their sums, C-mereology does not seem to be a good fit. The material constituents of a flame or any other bounded dissipative structure are continuously changing as more wax molecules interact with more oxygen molecules drawn in to the flame, but at each spatial location the same reactions are occurring. The products, mainly carbon dioxide and water pass out of the flame. Let us call these molecules 'fleeting 
parts'. A flame is a Whole of which the Parts are processes. How does the necessity for fleeting parts fit with the necessity for a process ontology? This problem has been addressed in the past. A rainbow is an optical phenomenon produced by the refraction and internal refection of light from a point source in rain drops. Theodoric of Freiburg, in his experimental study of the phenomenon, realised that he could treat the rain shower as a dissipative structure, because the rain drops were succeeding one another in the rain shower sufficiently quickly to allow for the modelling of the shower by a stationary array of watery spheres. The rain drops are fleeting parts of a dissipative structure. S-mereology seems to fit the concept of fleeting constituent well. There is a many membered set of raindrops at a certain location in the shower - just as there is a set of oxygen atoms at certain location in the flame. It is this set that is a constituent as a subset of the superset that is the flame.

Chemistry also makes use of 'ephemeral' individuals as parts of wholes. For instance, the swiftly composing and decomposing hydrogen-oxygen structures of which real water is really composed are ephemeral individuals. Water is made up of these beings. As such they are constituents of a certain whole. Here is another mereological set-up for which neither C-mereology nor S-mereology seems well adapted as discourse grammars. 


\section{References}

Earley, J. (2005) 'Why there is no salt in the sea' Foundations of Chemistry $785-102$.

Goodman, N. (1951) The Structure of Appearance. Cambridge MA: Harvard University Press.

Harré, R. (1996) From observability and manipulability: the inductive arguments for realism'. Synthese, $108137-155$.

Kistler, M. \& Gnassounou, B. (2007) Dispositions and Causal Powers. Aldershot: Ashgate. 181194.

Lewis, D. (1991) Parts of Classes. Oxford: Blackwell.

Llored, J.-P. (2009) Personal communications in "Le rôle de Mulliken dans l'Emergence de l'approximation des orbitales moléculaires", Master degree, University Paris X Nanterre (2007) ; "Chimie quantique et émergence", current Ph D work, Ecole Polytechnique and Free University of Brussels.

Mulliken, R. S. (1981) Life of a Scientist. Berlin and London: Springer-Verlag.

R.S. Mulliken (1932), “ Electronic structures of polyatomic molecules and valence II. General consideration”, Phys. Rev. 41, 50.

R.S. Mulliken (1933), "Electronic structures of polyatomic molecules and valence IV. Electronic states, quantum theory of the double bond". Phys .Rev. 43, 279-280.

R.S. Mulliken (1931), "Bonding power of electrons and theory of valence", Chem. Rev. 9, 350 .

Needham, P. (2005) 'Mixtures and modality'. Foundations of Chemistry. $7103-118$.

Ramsay, D. A. and Hinze, J. (1932) Selected Papers of Robert S. Mulliken.

Rescher, N. \& Oppenheimer, (1955)

Simons, P. (1987) Parts. Oxford: Clarendon Press. 